\title{
Diversité de l'ichtyofaune de la rivière Djerem : impact du barrage de Mbakaou et enjeux pour la conservation des poissons dans le Parc National du Mbam et Djerem (Cameroun)
}

\author{
Arnold Roger BITJA NYOM ${ }^{1,2, *}$, Samuel Didier NJOM ${ }^{1}$, Hermann Igor KITIO ${ }^{1}$ et \\ Charles Félix BILONG BILONG ${ }^{3}$
}

\author{
${ }^{I}$ Département de Sciences Biologiques, Faculté des Sciences, Université de Ngaoundéré, BP 454, Ngaoundéré, \\ Cameroun. \\ ${ }^{2}$ Département de Gestion des Pêches et Ecosystèmes Aquatiques, Institut des Sciences Halieutiques, Université \\ de Douala, BP 2701, Douala, Cameroun. \\ ${ }^{3}$ Département de Biologie et Physiologie Animales, Université de Yaoundé 1, BP 812 Yaoundé, Cameroun. \\ *Auteur correspondant ; E-mail : bitja.nyom_arnold@ymail.com; Tél : (+237) 677354075
}

\section{RESUME}

L'anthropisation intensive du bassin de la Sanaga impose une accélération des recherches sur la biodiversité et la conservation de ses ressources aquatiques. Dans ce contexte, l'ichtyofaune d'un segment du fleuve Djerem a été étudiée en vue d'identifier les déterminants environnementaux de sa distribution et de sa conservation. Trois stations situées respectivement en amont de la retenue d'eau de Mbakaou, au milieu de ce réservoir et en aval dans le Parc National du Mbam et Djérem (PNMD) ont été retenues pour ce travail. A la suite de l'échantillonnage par pêche d'avril à octobre 2015, l'étude taxonomique des poissons et de leurs habitats a révélé la présence de 44 espèces appartenant à 28 genres et à 13 familles. L'ichtyofaune du PNMD s'est montrée la plus diversifiée (33 espèces), dépourvue d'espèces invasives, avec une prédominance d'invertivores $(50,3 \%)$ et de pondeurs sur substrats divers (60,9\%). Celle du réservoir de Mbakaou a été la moins diversifiée (12 espèces), avec quatre espèces introduites devenues invasives et une prédominance d'herbivores / détritivores $(65,9 \%)$. Onze espèces endémiques ont suscité un intérêt pour d'éventuels programmes de conservation des poissons dans le PNMD à cause de leurs habitats restreints et sensibles tandis que 13 autres, toujours endémiques et connues du bassin de la Sanaga, n'ont pas été retrouvées dans la zone étudiée.

(C) 2020 International Formulae Group. All rights reserved.

Mots clés : Sanaga supérieur, poissons, espèces endémiques, espèces invasives, habitats, gestion.

\section{Diversity of the Djerem River ichthyofauna: impact of Mbakaou regulation dam and challenges for fish conservation in the Mbam and Djerem National Park (Cameroun)}

\begin{abstract}
The intensive anthropization of the Sanaga river basin requires to accelerate research on biodiversity and conservation of its aquatic resources. In this context, the ichthyofauna of a part of the Djerem River has been studied with the aim to know its diversity and identify environmental factors that determine its distribution and
\end{abstract}


conservation. Three stations were sampled from April to October 2015, they were located respectively upstream of the Mbakaou water reservoir, in the Mbakaou reservoir and downstream in the Mbam et Djerem National Park (PNMD). The Fish inventory followed by a taxonomic study and that of their habitats revealed the presence of 44 species belonging to 28 genera and 13 families. The ichthyofauna of PNMD with highest specific richness (33 species) was also devoid of invasive species, with a predominance of invertivorous $(50,3 \%$ ) and substratum egg-scatters $(60,9 \%)$. The ichtyofauna of the reservoir with lowest specific richness (12 species) contained also four alien species, with a predominance of herbivores / detritivores (65,9\%). 11 endemic species were concerned by the possible fish conservation programs in the PNMD due to their restricted and sensitive habitats while 13 other endemic species known from the Sanaga River basin were wanted in the study area.

(C) 2020 International Formulae Group. All rights reserved.

Keywords: Upper Sanaga, fish, endemic species, invasive species, habitats, management.

\section{INTRODUCTION}

Les menaces sur les poissons d'eaux douces d'Afrique sont, entre autres, la pêche intensive des espèces à faible résilience, les captures excessives pour l'aquariophilie et le commerce d'exportation, l'introduction d'espèces exotiques hautement compétitrices ou invasives, la pollution par déversement des polluants d'usines ou des plantations dans les cours d'eaux, la déforestation, les changements climatiques et les barrages des cours d'eaux (De Mérona, 2002 ; Lévêque et Paugy, 2006).

L'impact des barrages sur les peuplements de poissons est un sujet bien documenté. Pour de nombreux auteurs, les barrages auraient plus d'effets négatifs que positifs sur les peuplements de poissons. Parmi les effets négatifs, on cite la réduction ou la perte de biodiversité et d'habitats aquatiques, les obstacles physiques aux migrations longitudinales de poissons (à l'origine d'une rupture de connectivité piscicole entre les populations en amont et celles en aval) et la prolifération d'espèces introduites fortement compétitrices devenant invasives au détriment des espèces endémiques. L'effet positif le plus détectable est un meilleur développement du potentiel de pêche locale (Lévêque et Paugy, 2006).

$\mathrm{Au}$ Cameroun, le bassin du fleuve Sanaga occupe le premier rang puisqu'il draine à lui seul plus du quart de la superficie du pays (Bitja Nyom, 2015 ; Amougou et al., 2015). L'étude hydrologique de la Sanaga a permis de la diviser en trois grands segments contigus qui sont : la Sanaga supérieure, la Sanaga moyenne et la Sanaga inférieure. La Sanaga supérieure est représentée par le Djerem (son principal affluent) et le Lom, tous deux issus des monts $\mathrm{du}$ plateau de l'Adamaoua (Lienou et al., 2005; Onana, 2018). Plus long fleuve du Cameroun, la Sanaga revêt une importance capitale pour le pays sur les plans socioéconomique (de nombreuses pêcheries, sablières, fermes agricoles et industries se sont développées le long de ce fleuve et sont à l'origine d'une immigration suivie d'un brassage important d'exploitants des ressources en jeu), culturel (les peuples riverains ont développé une culture axée sur l'usage des produits de l'eau, le poisson occupant une place de choix dans les habitudes culinaires et médicinales) et hydroélectrique car de nombreux barrages y sont construits ou en voie de construction (Ako et al., 2010; Tchawa, 2012).

Du point de vue hydroélectrique, le cours de la Sanaga est parsemé de nombreuses chutes et de rapides importants qui justifient la construction de plusieurs barrages réservoirs et/ou hydroélectriques (Edéa en 1949 et Song Loulou en 1976 sur la Sanaga inférieure, Mbakaou en 1969 sur le Djerem, Bamendjing en 1974 sur le Noun, Mapé en 1987 sur le Mbam, Lom-Pangar en 2015 en aval de la confluence du Lom et du Pangar, Nachtigal en chantier depuis 2019) (Oreade-Breche, 2011 ; Bitja Nyom, 2015).

L'impact du barrage de Mbakaou sur la diversité des poissons du Djerem reste surtout documentée dans une littérature grise non publiée. Quelques études menées comme celles de Fotso et al. (2005) et de Dadem Gueguim (2011) ont plus concerné la socio-économie de 
la pêche. Elles ont montré que cette dernière constituait l'activité économique majeure des riverains en procurant les aliments et les emplois à plus d'un millier de personnes, avec une production annuelle estimée à 6000 tonnes de poissons pour une superficie d'exploitation d'environ 20600 ha (Fotso et al., 2005). La pêche est également pratiquée dans le Parc National du Mbam et Djerem (PNMD) situé en aval du barrage. Elle y est réglementée par une Convention de cogestion participative avec les riverains, laquelle délimite cette activité dans une partie du parc (Miyéré-Guirim) et fixe deux périodes ouvertes chaque année, du 1er avril au 30 juin et du $1^{\text {er }}$ octobre au 31 décembre tandis que les périodes de fermeture à la pêche courent du 1er janvier au 30 mars et du 1er juillet au 30 septembre de la même année (MINFOF, 2007).Une synthèse des travaux suscités indique qu'une trentaine d'espèces de poissons ont été répertoriées dans le Djerem ces 15 dernières années (OreadeBreche, 2011 ; Fosso et al., 2014). La présence du PNMD en aval du barrage de Mbakaou a motivé quelques-unes de ces études (Fosso et al., 2014). Cependant l'impact de cet édifice sur la diversité ichtyologique et les enjeux conséquents pour la conservation des poissons dans le PNMD sont encore des questions irrésolues.

Le but de ce travail était d'étudier la diversité des poissons du Djerem de part et d'autre du barrage de retenue de Mbakaou et dans le PNMD, puis déterminer les paramètres environnementaux qui régulent la distribution des espèces afin d'identifier les principaux enjeux pour la conservation des poissons dans cette zone d'étude.

\section{MATERIEL ET METHODES \\ Zone d'étude}

La zone d'étude retenue pour ce travail correspond au segment de la rivière Djerem situé entre le village Bétara (dans l'arrondissement de Ngaoundal) en amont et le Parc National du Mbam et Djerem (PNMD) en aval (Figure 1). A la suite d'une visite de prospection réalisée en mars 2015, trois stations ont été sélectionnées pour un échantillonnage réalisé en avril (période de transition entre la saison sèche et la saison de pluies) et en octobre (saison de pluies) de la même année. Il s'est agi de :

- la station amont (AM) située dans l'arrondissement de Ngaoundal en amont de la queue de la retenue d'eau de Mbakaou, plus précisément au sud du village Bétara, au lieudit Port de Fang-roum $\left(06^{\circ} 30^{\prime} 43^{\prime}\right.$ ' N, $013^{\circ}$ 04' 47,8' E). Durant ces campagnes de prélèvements, 25 pêcheurs y débarquaient régulièrement ;

- la station de la retenue d'eau de Mbakaou ou réservoir (RE). Les prélèvements ont été réalisés le long du réservoir sur un linéaire d'environ $10 \mathrm{~km}$ comprenant trois sites d'échantillonnage codés RE-1 ( $6^{\circ} 23^{\prime} 50,2^{\prime \prime} \mathrm{N}$, $\left.12^{\circ} 46^{\prime} 51,5^{\prime \prime} \mathrm{E}\right), \mathrm{RE}-2\left(06^{\circ} 21^{\prime} 31,1^{\prime \prime} \mathrm{N}, 012^{\circ}\right.$ $47^{\prime} 56,3^{\prime \prime}$ E) et RE-3 (06 $18^{\circ} 22,3^{\prime \prime} \mathrm{N}, 012^{\circ} 48^{\prime}$ $30,1 " \mathrm{E})$. Ce réservoir était exploité par environ 1600 pêcheurs qui capturaient environ 3533 tonnes de poissons par an (Dadem Gueguim, 2011) ;

- la station aval (AV) située dans le PNMD, près du lieu-dit Campement Débiro $\left(06^{\circ} 10^{\prime} 01^{\prime \prime} \mathrm{N}, 012^{\circ} 50^{\prime} 33,5^{\prime \prime} \mathrm{E}\right)$. Un nombre de 85 pêcheurs y prélevaient environ 171,360 tonnes de poissons par an (Dadem Gueguim, 2011).

\section{Mesure des variables environnementales}

Les paramètres physico-chimiques de l'eau de surface (température, $\mathrm{pH}$, conductivité électrique, solides totaux dissous ou TDS) de chaque station considérée ont été mesurés entre 7 et $8 \mathrm{~h}$ du matin, au moins à trois points différents, à l'aide d'un multi-paramètre de marque PCSTestr avant chaque échantillonnage.

\section{Pêche et conservation des échantillons de poissons}

L'échantillonnage des poissons a été fait en suivant les pêcheurs dans leurs sites de captures durant leurs sorties de pêche. Différents engins de pêche dormants (filets maillants de mailles variant entre 30 et $50 \mathrm{~mm}$, palangres de surface et de fond) ont été posés le soir à $18 \mathrm{~h}$, visités le lendemain matin à $6 \mathrm{~h}$, ensuite relevés le soir du même jour à $18 \mathrm{~h}$, ainsi continuellement de façon à réaliser une 
pêche nocturne et diurne de $72 \mathrm{~h}$ par station et par campagne. Les spécimens capturés ont été numérotés, photographiés sur le côté gauche, pesés au gramme près sur une balance électronique de marque Denver Instrument; leurs longueurs standard et totale ont ensuite été mesurées à l'aide d'un ichtyomètre. Une incision longitudinale de la paroi abdominale a été réalisée afin de permettre une bonne diffusion dans la carcasse du formaldéhyde dilué à $10 \%$, utilisé pour fixer les spécimens. $\mathrm{Au}$ laboratoire, après élimination du formaldéhyde par lavage abondant à l'eau de robinet, changée matin et soir pendant cinq jours, les spécimens fixés ont été conservés dans des bocaux contenant de l'alcool dilué à $70^{\circ}$.

\section{Identification des spécimens de poissons}

Les spécimens de poissons conservés ont été étudiés morphologiquement par des mensurations et des comptages classiques puis identifiés à l'aide des clés de Stiassny et al. (2007) et des révisions subséquentes impliquant notre zone d'étude (Lavoué, 2011 ; Vreven et al., 2016 ; Froese et al., 2019). La proximité géographique du Djerem (tête de bassin de la Sanaga en ichtyorégion de basse Guinée) d'avec les bassins du nord (Bénoué et Logone en ichtyorégion d'Afrique de l'Ouest) a autorisé la validation de nos identifications à l'aide des clés de Paugy et al. (2003).

\section{Analyse des données}

Les paramètres physico-chimiques mesurés ont été rendus par station et par mois (saison), les données des différents points d'échantillonnage d'une même station ayant été agglomérées. Le test-U de Mann Whitney a été effectué pour comparer les paramètres deux à deux entre stations et entre mois (saisons). Les différences des comparaisons statistiques ont été considérées significatives pour $\mathrm{P}<0,05$.

Les communautés de poissons des différentes stations ont été caractérisées par différents indices de diversité suivants: la richesse spécifique ou le nombre total d'espèces identifiées dans une station, l'indice de similarité de Sørensen $(S)$ utilisé pour apprécier les ressemblances/dissemblances éventuelles entre stations, l'indice de ShannonWeaver $\left(H^{\prime}\right)$ permettant d'évaluer la diversité des espèces composant le peuplement de chaque station en tenant compte des abondances spécifiques, enfin l'indice d'équitabilité de Pielou $(J)$ qui a servi à mesurer l'équirépartition des espèces du peuplement de chaque station par rapport à une répartition théorique égale de celles-ci (Zhang et Grabchak, 2016).

Les habitats d'alimentation et de reproduction des poissons inventoriés ont été déterminés au niveau générique ou spécifique à partir de la littérature lorsque disponible (Paugy et Lévêque, 2006 ; Froese et Pauly, 2019). Chaque espèce a été classée dans l'un des groupes trophiques suivants : herbivores / détritivores (HER), invertivores (INV), omnivores (OMV) ou piscivores (PIS) selon Paugy et Lévêque (2006) et Ibanez et al. (2007), et dans l'un des groupes de reproducteurs à savoir: les pondeurs en eau libre ou sur substrats divers incluant le sable, le gravier, les rochers et les végétaux (SUB), les gardiens d'œufs dans les nids de sable, de plantes ou d'écume (NES) et les incubateurs buccaux (INB) selon Paugy et Lévêque (2006) et Froese et Pauly (2019). La fréquence (\%) de chaque groupe trophique ou reproducteur a été calculée par station; elle a été le produit du nombre d'espèces du groupe considéré X 100 puis divisé par le nombre total d'espèces inventoriées dans cette station (Ibanez et al. 2007).

Les relations entre caractéristiques environnementales des habitats et l'ichtyofaune ont été évaluées par l'Analyse Canonique de Correspondance (CCA) couplée au test de Monte Carlo (1000 permutations) sous CANOCO Version 5.12 (Ter Braak et Šmilauer, 2012). 


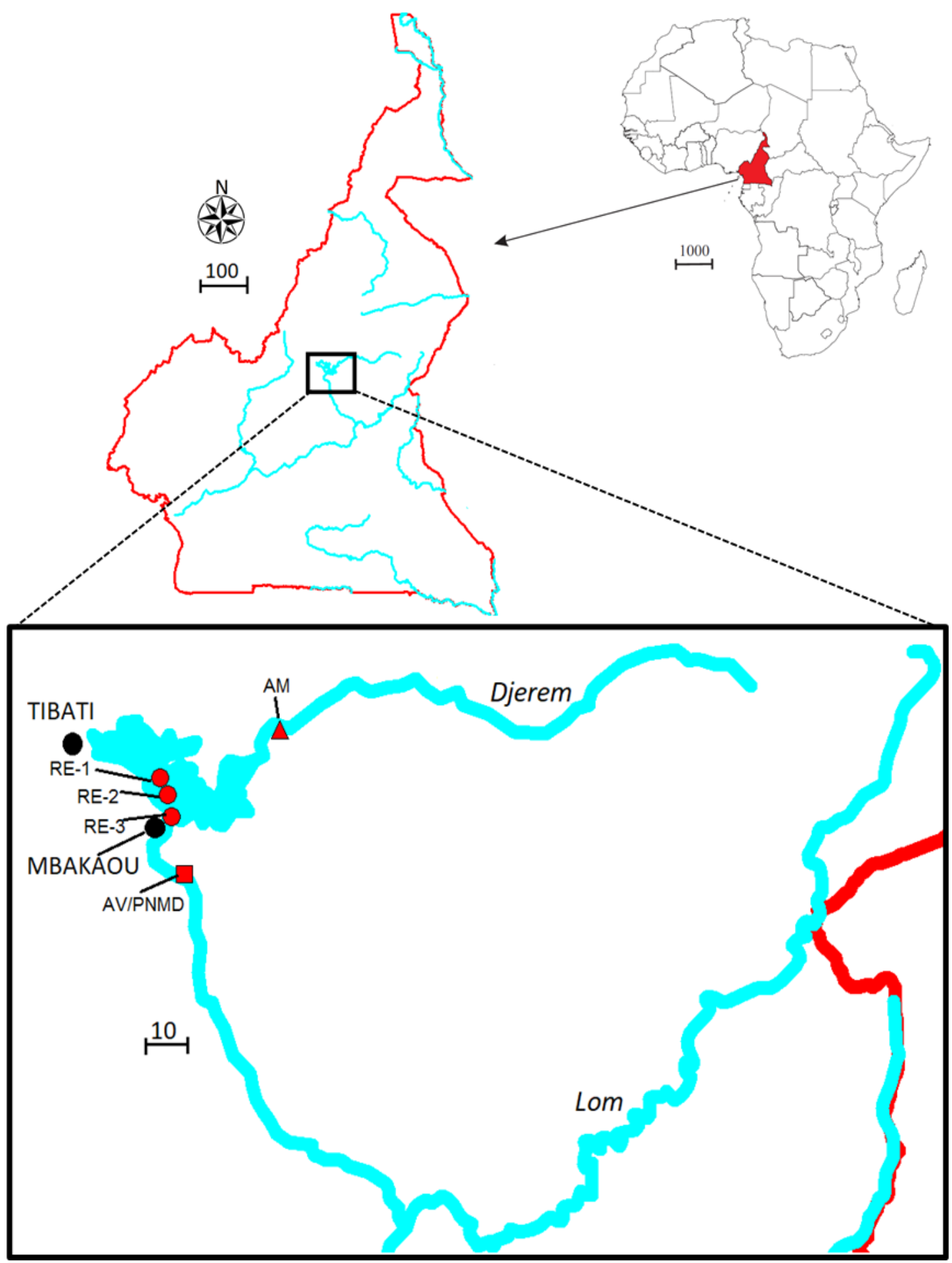

Figure 1 : Carte de localisation des sites d'étude dans le Djerem. AM : station amont ; RE : station du réservoir incluant trois points d'échantillonnage (RE-1, RE-2 et RE-3); AV : station aval située dans le Parc National du Mbam et Djerem (PNMD). 


\section{RESULTATS}

\section{Caractéristiques physico-chimiques de l'eau}

La température de l'eau a significativement été plus élevée $(\mathrm{P}<0.05)$ en avril qu'en octobre dans chacune des trois stations (Tableaux 1 et 2). Les différences entre stations durant le même mois n'étaient pas significatives, exceptée celle enregistrée en octobre entre les stations amont et aval.

En général, le pH s'est révélé relativement plus élevé en avril qu'en octobre à chacune de trois stations, les différences inter-mois n'étant significatives qu'en aval (Tableaux 1 et 2). Les différences entre stations des moyennes calculées ont été significatives en avril entre l'amont et l'aval et entre le réservoir et l'aval d'une part et, d'autre part, en octobre entre l'amont et le réservoir et entre l'amont et l'aval.

La teneur des solides totaux dissous (TDS) a été plus élevée $(\mathrm{P}<0,05)$ en avril comparé à octobre dans les stations amont et aval (Tableaux 1 et 2). En octobre, la teneur de ce paramètre s'est égalisée $(\mathrm{P}>0,05)$ entre les trois stations.

Comme pour les TDS, dans la même station, la conductivité électrique n'a varié significativement $(P<0,05)$ entre avril et octobre qu'en amont et en aval. En octobre, les valeurs de ce paramètre ont été significativement similaires $(\mathrm{P}>0,05)$ entre l'amont et le réservoir et entre ce dernier et l'aval, mais différentes $(\mathrm{P}<0,05)$ entre l'amont et l'aval (Tableau 2).

\section{Composition et caractéristiques de l'ichtyofaune inventoriée}

$\mathrm{Au}$ total, 44 espèces appartenant à 28 genres et 13 familles de poissons ont été identifiées dans les captures de pêche de notre zone d'étude (Tableau 3). De manière non exclusive entre stations, 18 espèces ont été échantillonnées en amont de la queue du réservoir, 12 dans le lac de retenue de Mbakaou et 33 dans le PNMD en aval du barrage.

De plus, 11 espèces connues endémiques de la Sanaga ont été retrouvées au cours de ce travail, neuf d'entre-elles (Doumea sanaga, Petrocephalus similis, Campylomormyrus phantasticus, Marcusenius sanagaensis, Sanagia velifera, Labeo nunensis, Labeo sanagaensis, Distichodus kolleri, Mastacembelus sanagali) n'étant présentes que dans le PNMD et les deux autres (Synodontis rebeli et Coptodon cameronensis) plus ubiquistes vivant dans les trois stations (Tableau 3).

Quatre espèces introduites dans le bassin de la Sanaga (Clarias gariepienus, Lates niloticus, Coptodon rendalli, Oreochromis niloticus) étaient relativement plus abondantes dans la retenue d'eau de Mbakaou. Huit autres taxa parmi les 12 répertoriés dans le lac ont été reconnus autochtones et non endémiques (exemple: Hydrocynus forskahlii, Hemichromis elongatus et Clarias camerunensis). Aucune des quatre espèces invasives qui pullulaient dans le réservoir n'a été capturée dans le PNMD durant nos campagnes d'échantillonnage (Tableau 3).

Les valeurs de l'indice de Shannon ont montré que la diversité de l'ichtyofaune était plus élevée $\left(H^{\prime}=1,3\right)$ dans le PNMD en aval et plus faible dans le réservoir $\left(H^{\prime}=0,8\right)$; celles de l'indice de Piélou ont révélé une répartition semblable des espèces dans les trois stations ( $J=0,9$ dans tous les échantillons), sauf celui de la retenue où il a légèrement diminué à 0,7 en avril (Tableau 4). L'indice de Sørensen a montré une similarité ichtyofaunique bien plus élevée $(60 \%)$ entre l'amont et le réservoir (ces deux stations avaient plus d'espèces en commun) et progressivement plus faible entre l'amont et l'aval $(35,3 \%)$ et le réservoir et l'aval $(17,8 \%)$ (Tableau 4).

\section{Fréquences des groupes trophiques}

Le peuplement de poissons du réservoir était essentiellement constitué de consommateurs primaires herbivores/détritivores $(65,9 \%)$, suivis de piscivores $(17,3 \%)$, d'omnivores $(16,3 \%)$ et d'invertivores dans une moindre mesure $(0,5 \%)$ (Figure 2). En amont et en aval de notre zone d'étude, les espèces des quatre groupes trophiques ont été capturées. Les invertivores y étaient plus représentés (respectivement 34,8\% et 50,3\%), suivis des omnivores (respectivement $31,4 \%$ et $32,6 \%$ ), des herbivores $(23,9 \%$ et $14,6 \%)$; les piscivores y 
ont été moins représentés avec respectivement $8,7 \%$ et $3,8 \%$ des effectifs.

\section{Fréquences des groupes reproducteurs}

Dans l'ichtyofaune du Djerem, les pondeurs sur substrat ou en pleine eau étaient numériquement dominants dans chacune des stations échantillonnées, soit de l'amont vers l'aval : $76,2 \% ; 55,3 \%$ et $60,9 \%$. Dans le réservoir, les incubateurs buccaux se rangeaient au second rang tandis qu'en amont et en aval, cette position était plutôt occupée par les gardiens d'œufs dans les nids avec des fréquences respectives de $23,8 \%$ et $31,5 \%$.

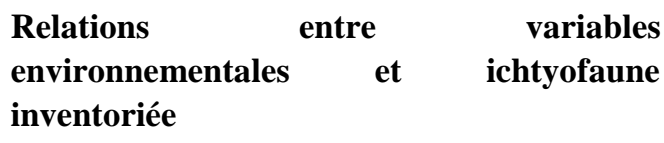

L'analyse canonique de correspondance (CCA) des paramètres environnementaux et de la composition de l'ichtyofaune a montré que les parties positives des axes 1 et 2 regroupaient les poissons du réservoir avec des fréquences élevées d'herbivores/détritivores (exemple : Labeo batesii, Labeo camerunensis, Coptodon cameronensis, Oreochromis niloticus), de piscivores (exemple: $H$. forskahlii, Lates niloticus et Hemichromis elongatus) et d'incubateurs buccaux $(O$. niloticus y étant très abondante). De plus, un nombre élevé de pêcheurs exploitaient cette ressource halieutique (Figure 4). La station aval, illustrée sur la Figure 4 par les parties négatives des axes 1 et 2, était caractérisée par son $\mathrm{pH}$ plus élevé en avril, ses habitats favorables aux pondeurs sur substrats divers et aux nicheurs, et sa richesse en invertivores et en omnivores. Le secteur amont correspondant au quadrant délimité par les parties négative et positive respectivement des axes 1 et 2 était caractérisé par des eaux de conductivité et de TDS relativement plus élevés, hébergeant des espèces telles que Paramormyrops kingsleyae et Brienomyrus brachyistius habituellement trouvées dans les rivières peu ou pas polluées.

Tableau 1 : Statistiques des paramètres physico-chimiques de l'eau exprimées sous forme de moyenne \pm écart-type.

\begin{tabular}{llllll}
\hline Stations & Mois & TEM $\left({ }^{\circ} \mathbf{C}\right)$ & $\mathbf{p H}$ & TDS $(\mathbf{p p m})$ & $\mathbf{C O N}\left(\boldsymbol{\mu s} \mathbf{~ c m}^{-\mathbf{1}}\right)$ \\
\hline Amont & Avril & $26,7 \pm 0,4$ & $5,7 \pm 0,2$ & $31,0 \pm 0,9$ & $44,4 \pm 1,1$ \\
& & & & & \\
\cline { 2 - 6 } & Octobre & $24,3 \pm 0,3$ & $5,9 \pm 0,3$ & $18,0 \pm 0,4$ & $26,3 \pm 0,2$ \\
\hline Réservoir & Avril & $26,8 \pm 0,3$ & $5,6 \pm 0,3$ & $16,6 \pm 5,2$ & $23,8 \pm 8,5$ \\
\cline { 2 - 6 } & Octobre & $24,8 \pm 0,3$ & $5,2 \pm 0,2$ & $17,3 \pm 2,3$ & $24,8 \pm 2,7$ \\
\hline Aval & Avril & $26,6 \pm 0,1$ & $6,1 \pm 0,1$ & $23,5 \pm 0,5$ & $30,6 \pm 2,0$ \\
& & & & & \\
\cline { 2 - 6 } & Octobre & $25,2 \pm 0,3$ & $5,0 \pm 0,1$ & $16,4 \pm 0,4$ & $23,0 \pm 0,3$ \\
\hline
\end{tabular}

CON : Conductivité électrique ; TEM : Température ; TDS : Solides totaux dissous 
Tableau 2 : Valeurs de $P$ obtenues du test-U de Mann Whitney (MW) de comparaisons par paire suite au test de Kruskal Wallis $\mathrm{P}<0,05$ dans tous les cas.

\begin{tabular}{|c|c|c|c|c|c|c|c|c|}
\hline & & & \multicolumn{2}{|c|}{$\mathbf{A M}$} & \multicolumn{2}{|c|}{$\mathbf{R E}$} & \multicolumn{2}{|c|}{$\mathbf{A V}$} \\
\hline & & & Avr & Oct & Avr & Oct & Avr & Oct \\
\hline \multirow{6}{*}{ Temperature } & \multirow{2}{*}{$\mathrm{AM}$} & Avr & & & & & & \\
\hline & & Oct & $0,008 *$ & & & & & \\
\hline & \multirow{2}{*}{$\mathrm{RE}$} & Avr & 0,540 & $0,008 *$ & & & & \\
\hline & & Oct & $0,008 *$ & 0,063 & $0,008 *$ & & & \\
\hline & \multirow{2}{*}{$\mathrm{AV}$} & Avr & 0,952 & $0,012 *$ & 0,357 & $0,012 *$ & & \\
\hline & & Oct & $0,008^{*}$ & $0,008 *$ & $0,008 *$ & 0,127 & $0,012 *$ & \\
\hline \multirow{6}{*}{$\mathrm{pH}$} & \multirow{2}{*}{$\mathrm{AM}$} & Avr & & & & & & \\
\hline & & Oct & 0,206 & & & & & \\
\hline & \multirow{2}{*}{$\mathrm{RE}$} & Avr & 0,889 & 0,206 & & & & \\
\hline & & Oct & $0,024 *$ & $0,012 *$ & 0,103 & & & \\
\hline & \multirow{2}{*}{$\mathrm{AV}$} & Avr & $0,016^{*}$ & 0,667 & $0,008^{*}$ & $0,008^{*}$ & & \\
\hline & & Oct & $0,008^{*}$ & $0,012 *$ & $0,016^{*}$ & 0,167 & $0,008 *$ & \\
\hline \multirow{6}{*}{ TDS } & \multirow{2}{*}{$\mathrm{AM}$} & Avr & & & & & & \\
\hline & & Oct & $0,008 *$ & & & & & \\
\hline & \multirow{2}{*}{$\mathrm{RE}$} & Avr & $0,008^{*}$ & 0,341 & & & & \\
\hline & & Oct & $0,008 *$ & 0,595 & 0,738 & & & \\
\hline & \multirow{2}{*}{ AV } & Avr & $0,008 *$ & $0,008 *$ & 0,151 & $0,008 *$ & & \\
\hline & & Oct & $0,012^{*}$ & $0,012 *$ & 0,794 & 0,548 & $0,012 *$ & \\
\hline \multirow{6}{*}{ Conductivité } & \multirow{2}{*}{$\mathrm{AM}$} & Avr & & & & & & \\
\hline & & Oct & $0,012^{*}$ & & & & & \\
\hline & \multirow{2}{*}{$\mathrm{RE}$} & Avr & $0,008 *$ & 0,651 & & & & \\
\hline & & Oct & $0,008 *$ & 0,127 & 0,690 & & & \\
\hline & \multirow{2}{*}{ AV } & Avr & $0,008 *$ & $0,012 *$ & 0,421 & $0,008^{*}$ & & \\
\hline & & Oct & $0,008 *$ & $0,012 *$ & 0,690 & 0,151 & $0,008 *$ & \\
\hline
\end{tabular}

AM: Amont ; AV : Aval; RE : Reservoir; Avr: Avril; Oct: Octobre

Tableau 3 : Familles et espèces de poissons inventoriées, leurs caractéristiques biogéographiques, alimentaires et reproductrices, leur statut à l'Union Internationale pour la Conservation de la Nature (UICN) selon la base de données consultée le 05/05/2020 et leur présence - absence dans les stations étudiées.

\begin{tabular}{|c|c|c|c|c|c|c|c|c|c|}
\hline \multirow[b]{2}{*}{ Familles } & \multirow[b]{2}{*}{ Espèces } & \multirow[b]{2}{*}{ Codes } & \multirow[b]{2}{*}{ CB } & \multirow[b]{2}{*}{ GT } & \multirow[b]{2}{*}{ GR } & \multirow{2}{*}{$\begin{array}{l}\text { Statut } \\
\text { IUCN }\end{array}$} & \multicolumn{3}{|c|}{ Stations } \\
\hline & & & & & & & $\mathbf{A M}$ & $\mathbf{R E}$ & $\mathbf{A V}$ \\
\hline \multirow[t]{3}{*}{ Alestidae } & Alestes macrophthalmus & Alemac & $\mathrm{N}$ & INV & SUB & LC & & & $\mathrm{x}$ \\
\hline & Brycinus macrolepidotus & Brymac & $\mathrm{N}$ & OMV & SUB & $\mathrm{LC}$ & $\mathrm{x}$ & & $\mathrm{x}$ \\
\hline & Hydrocynus forskahlii & Hydfor & $\mathrm{N}$ & PIS & SUB & $\mathrm{LC}$ & & $\mathrm{x}$ & $\mathrm{x}$ \\
\hline Amphiliidae & Doumea sanaga & Dousan & $\mathrm{E}$ & OMV & SUB & $\mathrm{LC}$ & & & $\mathrm{x}$ \\
\hline Bagridae & Bagrus docmak & Bagdoc & $\mathrm{N}$ & PIS & SUB & $\mathrm{LC}$ & & & $\mathrm{x}$ \\
\hline Cichlidae & Coptodon cameronensis & Copcam & $\mathrm{E}$ & HER & NES & $\mathrm{LC}$ & $\mathrm{x}$ & $\mathrm{x}$ & $\mathrm{x}$ \\
\hline
\end{tabular}




\begin{tabular}{|c|c|c|c|c|c|c|c|c|c|}
\hline & Coptodon rendalli & Copren & $\mathrm{I}$ & HER & SUB & $\mathrm{LC}$ & $\mathrm{x}$ & $\mathrm{x}$ & \\
\hline & Hemichromis elongatus & Hemelo & $\mathrm{N}$ & PIS & NES & $\mathrm{LC}$ & $\mathrm{x}$ & $\mathrm{x}$ & \\
\hline & Oreochromis niloticus & Orenil & I & HER & INB & $\mathrm{LC}$ & $\mathrm{x}$ & $\mathrm{x}$ & \\
\hline \multirow[t]{5}{*}{ Clariidae } & Clarias camerunensis & Clacam & $\mathrm{N}$ & OMV & SUB & $\mathrm{LC}$ & $\mathrm{x}$ & & $\mathrm{x}$ \\
\hline & Clarias gariepinus & Clagar & I & OMV & SUB & $\mathrm{LC}$ & $\mathrm{x}$ & $\mathrm{x}$ & \\
\hline & Clarias jaensis & Clajae & $\mathrm{N}$ & OMV & SUB & $\mathrm{LC}$ & $\mathrm{x}$ & & $\mathrm{x}$ \\
\hline & Clarias longior & Clalon & $\mathrm{N}$ & OMV & SUB & $\mathrm{LC}$ & $\mathrm{x}$ & & \\
\hline & Clarias pachynema & Clapac & $\mathrm{N}$ & OMV & SUB & $\mathrm{LC}$ & & & $\mathrm{x}$ \\
\hline \multirow[t]{2}{*}{ Claroteidae } & Chrysischtys auratus & Chraur & $\mathrm{N}$ & INV & SUB & $\mathrm{LC}$ & & & $\mathrm{x}$ \\
\hline & Parauchenoglanis balayi & Parbal & $\mathrm{N}$ & INV & SUB & $\mathrm{LC}$ & $\mathrm{x}$ & $\mathrm{x}$ & $\mathrm{x}$ \\
\hline \multirow[t]{9}{*}{ Cyprinidae } & Enteromius guirali & Entgui & $\mathrm{N}$ & OMV & SUB & $\mathrm{LC}$ & $\mathrm{x}$ & $\mathrm{x}$ & \\
\hline & Labeo batesii & Labbat & $\mathrm{N}$ & HER & SUB & $\mathrm{LC}$ & & $\mathrm{x}$ & \\
\hline & Labeo camerunensis & Labcam & $\mathrm{N}$ & HER & SUB & $\mathrm{LC}$ & & $\mathrm{x}$ & \\
\hline & Labeo nunensis & Labnun & $\mathrm{E}$ & HER & SUB & $\mathrm{LC}$ & & & $\mathrm{x}$ \\
\hline & Labeo sanagaensis & Labsan & $\mathrm{E}$ & HER & SUB & $\mathrm{LC}$ & & & $\mathrm{x}$ \\
\hline & Labeobarbus brevispinis & Labbre & $\mathrm{N}$ & HER & SUB & $\mathrm{LC}$ & & & $\mathrm{x}$ \\
\hline & Labeobarbus habereri & Labhab & $\mathrm{N}$ & HER & SUB & $\mathrm{LC}$ & & & $\mathrm{x}$ \\
\hline & Labeobarbus mariae & Labmar & $\mathrm{N}$ & HER & SUB & $\mathrm{LC}$ & & & $\mathrm{x}$ \\
\hline & Sanagia velifera & Sanvel & $\mathrm{E}^{*}$ & HER & SUB & NT & & & $\mathrm{x}$ \\
\hline Distichodontidae & Distichodus kolleri & Diskol & $\mathrm{E}$ & HER & SUB & $\mathrm{LC}$ & & & $\mathrm{x}$ \\
\hline Latidae & Lates niloticus & Latnil & $\mathrm{I}$ & PIS & SUB & $\mathrm{LC}$ & $\mathrm{x}$ & $\mathrm{x}$ & \\
\hline Mastacembelidae & Mastacembelus sanagali & Massan & $\mathrm{E}$ & INV & NES & $\mathrm{LC}$ & & & $\mathrm{x}$ \\
\hline Mochokidae & Synodontis rebeli & Synreb & $\mathrm{E}$ & OMV & SUB & DD & $\mathrm{x}$ & $\mathrm{x}$ & $\mathrm{x}$ \\
\hline \multirow[t]{14}{*}{ Mormyridae } & Brienomyrus brachyistius & Bribra & $\mathrm{N}$ & INV & SUB & $\mathrm{LC}$ & $\mathrm{x}$ & & \\
\hline & Campylomormyrus phantasticus & Campha & $\mathrm{E}$ & INV & NES & $\mathrm{LC}$ & & & $\mathrm{x}$ \\
\hline & Hippopotamyrus castor & Hipcas & $\mathrm{N}$ & INV & NES & $\mathrm{LC}$ & & & $\mathrm{x}$ \\
\hline & Paramormyrops kingsleyae & Parkin & $\mathrm{N}$ & INV & NES & DD & $\mathrm{x}$ & & \\
\hline & Marcusenius mento & Marmen & $\mathrm{N}$ & INV & NES & $\mathrm{LC}$ & $\mathrm{x}$ & & $\mathrm{x}$ \\
\hline & Marcusenius moorii & Marmoo & $\mathrm{N}$ & INV & NES & $\mathrm{LC}$ & & & $\mathrm{x}$ \\
\hline & Marcusenius sanagaensis & Marsan & $\mathrm{E}$ & OMV & SUB & VU & & & $\mathrm{x}$ \\
\hline & Mormyrops anguilloides & Morang & $\mathrm{N}$ & OMV & SUB & $\mathrm{LC}$ & $\mathrm{x}$ & & $\mathrm{x}$ \\
\hline & Mormyrops breviceps & Morbre & $\mathrm{N}$ & OMV & SUB & $\mathrm{LC}$ & & & $\mathrm{x}$ \\
\hline & Mormyrops caballus & Morcab & $\mathrm{N}$ & INV & NES & $\mathrm{NE}$ & & & $\mathrm{x}$ \\
\hline & Mormyrus macrophtalmus & Mormac & $\mathrm{N}$ & INV & NES & $\mathrm{LC}$ & & & $\mathrm{x}$ \\
\hline & Mormyrus rume & Morrum & $\mathrm{N}$ & INV & NES & $\mathrm{NE}$ & & & $\mathrm{x}$ \\
\hline & Mormyrus tapirus & Mortap & $\mathrm{N}$ & INV & SUB & $\mathrm{LC}$ & & & $\mathrm{x}$ \\
\hline & Petrocephalus similis & Petsim & $\mathrm{E}$ & INV & SUB & $\mathrm{NE}$ & $\mathrm{x}$ & & $\mathrm{x}$ \\
\hline Schilbeidae & Schilbe mytus & Schmys & $\mathrm{N}$ & OMV & SUB & $\mathrm{LC}$ & & & $\mathrm{x}$ \\
\hline
\end{tabular}

CB : caractéristiques biogéographiques ; GT : groupe trophique ; GR : groupe reproducteur ; * : présence possible dans le Nyong (Froese et Pauly, 2019).

$\mathrm{E}$ : endémique ; I : introduite ; $\mathrm{N}$ : Native-non endémique ; HER: herbivores/détritivores, INV: invertivores, OMV: omnivores, PIS: piscivores ; INB: incubateurs buccaux ; NES: gardiens d'œufs dans les nids d'écume, des trous, de sable ou des feuilles; SUB: pondeurs en milieux ouverts ou sur substrats de sable, gravier, rochers ou plantes ; Union Internationale pour la Conservation de la Nature (UICN) ; DD: Données insuffisantes; EN: En danger ; LC: Préoccupation mineure; NE: Non évaluée; NT: Quasi menacée; VU: Vulnérable ; présence (x) ; absence (vide) ; AM : amont ; AV : aval ; RE : réservoir. 
Tableau 4 : Valeurs de la richesse spécifique et des indices de diversité (Shannon, Piélou et Sørensen) par station et par mois d'échantillonnage dans la zone d'étude.

\begin{tabular}{|c|c|c|c|c|c|c|c|c|c|}
\hline \multirow{3}{*}{ Station } & \multirow{2}{*}{\multicolumn{2}{|c|}{$\begin{array}{l}\text { Richesse } \\
\text { spécifique }\end{array}$}} & \multicolumn{7}{|c|}{ Indices } \\
\hline & & & \multicolumn{2}{|c|}{ Shannon } & \multicolumn{2}{|c|}{ Piélou } & \multicolumn{3}{|c|}{ Sørensen (\%) } \\
\hline & Avr & Oct & Avr & Oct & Avr & Oct & AM/RE & $\mathrm{AM} / \mathrm{AV}$ & RE/AV \\
\hline $\mathrm{AM}$ & 11 & 17 & 0,9 & 1,1 & 0,9 & 0,9 & \multirow{3}{*}{60,0} & \multirow{3}{*}{35,3} & \multirow{3}{*}{17,8} \\
\hline$\overline{\mathrm{RE}}$ & 12 & 8 & 0,8 & 0,8 & 0,7 & 0,9 & & & \\
\hline AV & 28 & 21 & 1,3 & 1,2 & 0,9 & 0,9 & & & \\
\hline
\end{tabular}

AM: Amont ; AV : Aval; RE : Reservoir; Avr: Avril; Oct: Octobre

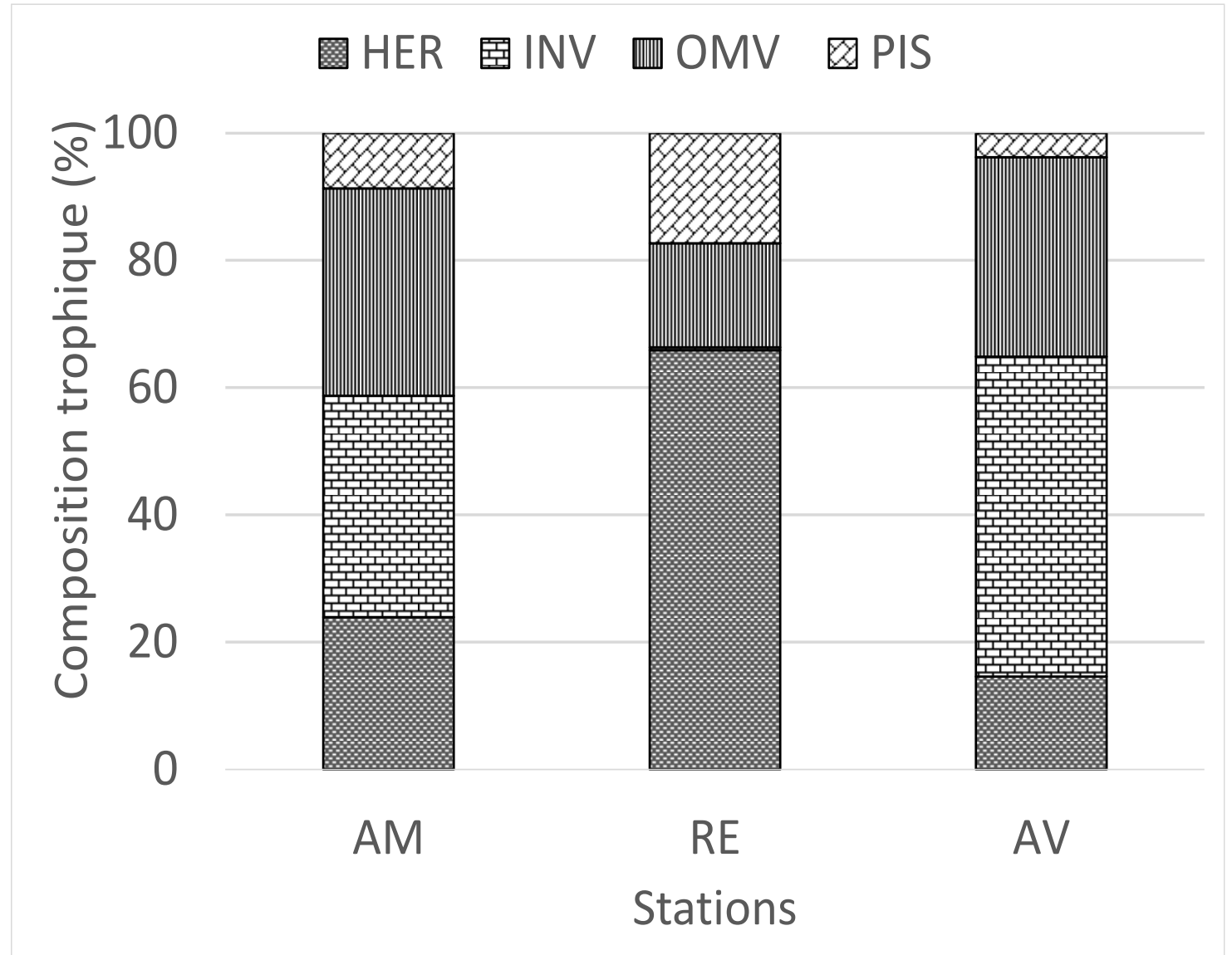

Figure 2 : Fréquences des groupes trophiques identifiés dans les trois stations.AM : amont, RE : réservoir, AV : aval, HER : herbivores / détritivores, INV : invertivores, OMV : omnivores, PIS : piscivores. 


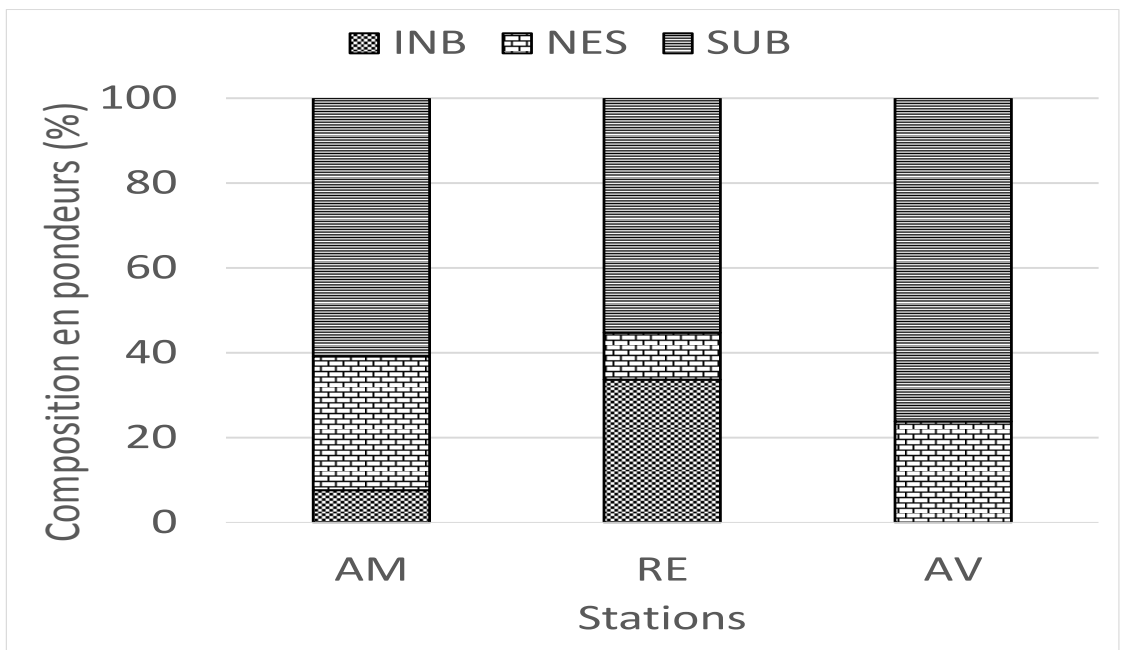

Figure 3 : Fréquences des groupes de pondeurs identifiés dans les trois stations. AM : amont, RE : réservoir, AV : aval; INB : incubateurs buccaux, NES : gardiens d'œufs dans les nids de sable, de plantes ou d'écume, SUB : pondeurs en eau libre ou sur substrats divers.

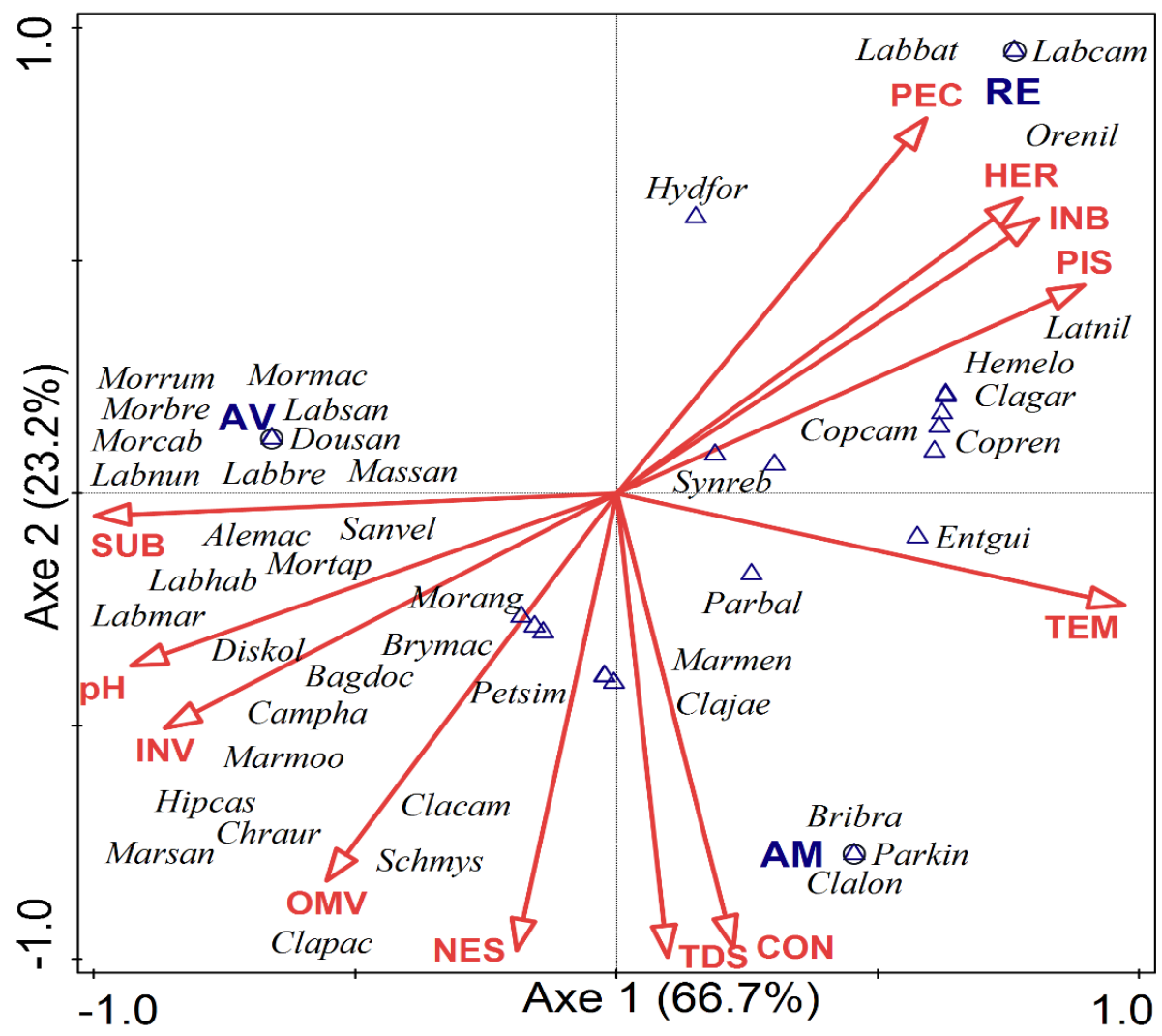

Figure 4: Diagramme de l'Analyse Canonique de Correspondance entre variables environnementales et ichtyofaune inventoriée. AM : amont, AV: aval, RE : réservoir ; CON : Conductivité électrique, PEC : nombre de pêcheurs par station, TDS : Solides totaux dissous, TEM : Température; HER : herbivores / détritivores, INV : invertivores, OMV : omnivores, PIS : piscivores ; INB : incubateurs buccaux, NES : gardiens d'œufs dans les nids de sable, de plantes ou d'écume, SUB : pondeurs en eau libre ou sur substrats divers. 


\section{DISCUSSION}

\section{Diversité des poissons dans la zone d'étude}

L'étude de l'ichtyofaune de trois stations d'un segment du Djerem mesurant environ $70 \mathrm{~km}$ (soit environ 20\% des $350 \mathrm{~km}$ de longueur du Djerem) a révélé la présence de $26,04 \%$ des 169 espèces d'eaux douces et saumâtres connues dans l'ensemble du bassin du fleuve Sanaga (Froese et Pauly, 2019). L'absence dans notre échantillon d'espèces de petite taille telles que les Cyprinodontiformes trouve sa justification dans la sélectivité des engins de pêches utilisés, elle-même conditionnée par la législation en vigueur concernant les mailles des filets $(>30 \mathrm{~mm})$ autorisées dans cette zone d'étude (MINFOF, 2007).

\section{Impact du barrage de Mbakaou sur la diversité des poissons}

Le réservoir de Mbakaou, qui a subi des variations saisonnières du $\mathrm{pH}$ et des teneurs en solides totaux dissous, a hébergé à un taux élevé des herbivores microphages et des détritivores indiquant une abondance de phytoplancton dans ce lac tandis que la fréquence élevée de piscivores en cette station suggère une disponibilité suffisante des proies. Cependant, la faible diversité ichtyofaunique observée dans ce réservoir, délimité en amont et en aval par deux stations ayant des diversités biologiques plus élevées, prouve à suffisance que le barrage de Mbakaou contribue à la diminution de la biodiversité locale. L'ensemble de nos résultats montre que ce barrage favorise la prolifération d'espèces étrangères incluant de redoutables ichtyophages comme Lates niloticus, de bons compétiteurs consommateurs primaires comme Oreochromis niloticus et Coptodon rendalli et des omnivores robustes comme Clarias gariepinus. Cette prolifération d'espèces introduites ayant une bonne résilience et atteignant des tailles intéressantes pour la pêche, attire à son tour un grand nombre de pêcheurs qui induisent une mortalité conséquente par une pêche intensive. L'effet cumulé de la prédation, de la compétition et de la pêche intensive représente un ensemble de menaces à l'origine de la diminution importante de la biodiversité observée dans le réservoir. De nombreux cas de baisse de diversité ichtyofaunique obéissant à ce schéma dans d'autres lacs de barrages en Afrique ont été rapportés dans la littérature (Lévêque et Paugy, 2006; Montchowui et al., 2008; Fagnon et al., 2013 ; Kamélan et al., 2013 ; Gbaguidi et al., 2016).

\section{Impact du barrage de Mbakaou sur la distribution des espèces}

Bien qu'aucune des quatre espèces invasives qui pullulent dans le réservoir n'a été retrouvée dans le PNMD durant la campagne de 2015, leur présence immédiatement en aval $\mathrm{du}$ barrage est hautement probable (Conservateur du PNMD, com. pers.). Une telle présence pourrait, en partie, procéder d'une diffusion progressive des œufs et des juvéniles dans le courant qui traverse les vannes de restitution d'eau vers l'aval. Ce phénomène a été observé dans d'autres dispositifs de prise d'eau à travers les barrages à l'échelle internationale (Sonny et al., 2006). Toutefois, la différence de composition ichtyofaunique entre le réservoir et la station aval du PNMD est remarquable dans ce travail ; la dispersion d'adultes vers le PNMD semble réduite par la pêche pratiquée au pied du barrage et probablement par la mosaïque d'habitats constitués selon Olivry (1986) d'une succession de chutes et de rapides (d'une dénivelée totale d'une cinquantaine de mètres) qui existent entre l'aval du barrage et le PNMD. Le contrôle et le suivi de la présence des espèces invasives dans le PNMD sont, de ce fait, fortement recommandés car si leur présence venait à être confirmée, leur élimination progressive par une pêche sélective se révèlerait souhaitable. 


\section{Origine des espèces invasives trouvées dans le Djerem}

L'absence ici constatée des espèces invasives dans le Djerem loin en aval du barrage, indique que celles-ci ne sont pas natives de ce cours d'eau. En effet, la littérature renseigne sur leurs origines probables. D'après Vreven et al. (2007), des stocks d'Oreochromis niloticus venant du Tchad et de la République Centrafricaine et ceux probablement constitués d'un mélange de Coptodon rendalli et de Coptodon zillii originaires de la République Démocratique du Congo auraient été introduits dans le bassin de la Sanaga, en particulier dans les marais du Noun, du Djerem et du Lom, entre 1949 (avant la construction du barrage de Mbakaou) et 1975 (après la mise en eau dudit barrage). Bien que Coptodon zillii n'a pas été identifiée lors de nos campagnes de 2015, sa présence probable dans notre zone d'étude est signalée dans la littérature. Ces introductions d'espèces étrangères dont certaines étaient accidentelles (Vreven et al., 2007), avaient pour but de développer l'aquaculture et la pêche, et d'améliorer la productivité des milieux naturels (Lévêque, 2006).

\section{Implications pour la conservation dans le PNMD}

Onze espèces sur les 44 identifiées dans notre zone d'étude étaient endémiques du bassin de la Sanaga, soit environ $46 \%$ des 24 taxa d'eaux douces considérés endémiques de ce bassin (Stiassny et al., 2007 ; Froese et Pauly, 2019). Ces espèces sensibles sont concernées pour la conservation (Moelants, 2010 ; Bitja Nyom, 2015). Ces onze taxa endémiques identifiés dans le PNMD n'ont point été retrouvés dans le réservoir, excepté deux (Synodontis rebelli et Coptodon cameronensis) qui montrent ainsi une meilleure résilience. Bien que les onze espèces endémiques aient été échantillonnées dans la Sanaga moyenne, où elles étaient potentiellement soumises aux mêmes pressions environnementales qu'à Mbakaou (Bitja
Nyom, 2015), leur pêche dans le PNMD devrait être contrôlée/réglementée. On limiterait ainsi l'impact éventuel des effets cumulatifs des pressions exogènes (baisse saisonnière de la qualité du courant d'eau descendant, compétition potentielle avec les espèces invasives échappées du réservoir de Mbakaou et ayant atteint le PNMD) et de la pêche autorisée saisonnièrement dans cette aire protégée sur l'ichtyofaune du parc.

L'une des espèces de poissons endémiques du bassin de la Sanaga et particulièrement caractéristique du Djerem, Schilbe djeremi (Thys van den Audenaerde \& De Vos, 1982), n'a pas été capturée lors de nos campagnes de 2015 ni signalée depuis de nombreuses années dans les récoltes de pêches de cette zone. Si elle a survécu, chose qu'il n'a pas été possible de confirmer en 2015, elle devrait être aussi prise en compte dans le programme de conservation des poissons du PNMD, au même titre que les 13 taxa ci-après cités, endémiques du bassin du fleuve Sanaga, susceptibles d'être présents dans le Djerem mais n'ayant pas été retrouvés lors de nos campagnes de 2015: Aphyosemion dargei Amiet, 1987; Chiloglanis sanagaensis Roberts, 1989 ; Chrysichthys longidorsalis Risch \& Thys van den Audenaerde, 1981; Enteromius bourdariei (Pellegrin, 1928); Labeobarbus fimbriatus (Holly, 1926); Labeobarbus jaegeri (Holly, 1930); Labeobarbus mbami (Holly, 1927); Mastacembelus seiteri Thys van den Audenaerde, 1972; Nannocharax rubrolabiatus Van den Bergh, Teugels, Coenen \& Ollevier, 1995; Notoglanidium depierrei (Daget, 1979); Prolabeops melanhypopterus (Pellegrin, 1928) et Sarotherodon galilaeus sanagaensis (Thys van den Audenaerde, 1966).

\section{Conclusion}

L'ichtyofaune $\mathrm{du}$ Djerem est très diversifiée et comporte un mélange d'espèces autochtones et d'espèces étrangères 
introduites. Ces dernières sont peu diversifiées mais abondantes et permettent le développement de la pêche à Mbakaou. Toutefois, elles induisent une baisse de diversité dans ce réservoir. Les barrages, la pollution, la surpêche et la perte d'habitats ne sont donc pas les seuls problèmes environnementaux posés par l'anthropisation du fleuve Sanaga; il y a aussi et surtout la prolifération d'espèces étrangères, initialement introduites dans ce bassin hydrographique dans le but de développer la pêche et l'aquaculture, qui aujourd'hui sont devenues invasives. Les parcs nationaux qui existent le long du fleuve Sanaga, à l'exemple du PNMD, devraient être mis à contribution pour davantage identifier et conserver l'ichtyofaune menacée.

\section{CONFLIT D'INTERETS}

Les auteurs attestent qu'il n'existe aucun conflit d'intérêts entre eux ou autre partie au sujet de cet article.

\section{CONTRIBUTIONS DES AUTEURS}

ARBN a conçu le dispositif expérimental ; $\mathrm{ABN}$, SDN, HIK et CFB ont menés des travaux d'échantillonnage sur le terrain; ARBN et SDN ont analysé les données; tous les auteurs ont participé à la rédaction de l'article.

\section{REMERCIEMENTS}

Les auteurs remercient le Conservateur du PNMD en 2015 (M. Albert Mounga Abana), les échogardes et pêcheurs (M. Edgar Désiré Ambassa Ongono, M. Ruffin Duplex Ambahé et M. Nicolas Nga Okali) pour leur hospitalité et leur aide lors de la collecte des données sur le terrain. Ils expriment également leur reconnaissance envers M. Bernard Fosso et M. David Nzouango de Wild Life Conservation Society (WCS) pour leurs conseils et la documentation mise à leur disposition.

\section{REFERENCES}

Ako AA, Eyong GET, Nkeng GE. 2010. Water Resources Management and Integrated Water Resources Management (IWRM) in Cameroon. Water Resources Management, 24: 871-888. DOI: https://doi.org/10.1007/s11269-0099476-4

Amougou JA, Ndam Ngoupayou JR, Djocgoué PF, Bessoh Bell S, Batha R. 2015. Variabilité climatique et régime hydrologique en milieu bioclimatique de transition : cas du bassin fluvial de la Sanaga. Afrique Science, 11 (5) : 51-77.

Bitja Nyom AR. 2015. Etat initial des peuplements piscicoles. Rapport final. http://documents.worldbank.org/curated/ pt/930081498550117079/pdf/116789-

V6-EA-FRENCH-P157734-

Box402916B-PUBLIC-Disclosed-6-262017.pdf

Dadem Gueguim C. 2011. Durabilité de la pêche le long du cours d'eau Djerem dans le Parc National du Mbam et Djerem et dans la retenue d'eau de Mbakaou. Mémoire de fin d'étude d'ingénierie, Université de Dschang, Dschang, p. 100.

De Mérona B. 2002. Dynamique des peuplements de poissons dans le réservoir de Petit-Saut (Fleuve Sinnamary, Guyane française). Bulletin Français de la Pêche et de la Pisciculture, 364 : 5-22. DOI : http://dx.doi.org/10.1051/kmae:2002001

Fagnon MS, Chikou A, Youssao I, Laleye P. 2013. Caractérisation morphologique des populations de Sarotherodon melanotheron (Pisces, Cichlidae) en eaux douces et saumâtres au Sud Bénin. Int. J. Biol. Chem. Sci., 7(2): 619-630. DOI: http://dx.doi.org/10.4314/ijbcs.v7i2.18

Fosso B, Nzouango D, Ambahe R. 2014. Projet d'appui à l'aménagement du Parc National du Mbam-Djerem. WCSCameroon Biodiversity Program : Mbakaou. 
Fotso R, Hiol F, Pouomegne J. 2005. Etude socio-économiques de la région du Mbam et Djerem. WCS-Projet d'Appui à l'Aménagement du Parc National du Mbam et Djerem : Mbakaou.

Froese R, Pauly D. 2019. FishBase. World Wide Web electronic publication. www.fishbase.org, version (12/2019).

Gbaguidi HMAG, Adite A, Sossoukpe E. 2016. Ecology and Fish Biodiversity of Man-Made Lakes of Southern Benin (West Africa): Implications for Species Conservation and Fisheries Management. Journal of Environmental Protection, 7: 874-894. DOI : http://dx.doi.org/10.4236/jep.2016.76079 Ibanez C, Oberdorff T, Teugels G, Mamononekene V, Lavoué S, Fermon Y, Paugy D, Toham AK. 2007. Fish assemblage structure and function along environmental gradients in rivers of Gabon (Africa). Ecology of Freshwater Fish, 16: 315-334. DOI: 10.1111/j.16000633.2006.00222.x.

Kamélan TM, Berté S, N'zi KG, Bamba M, Gooré Bi G, Kouamélan EP. 2013. Peuplement ichtyologique du complexe Brimé-Méné-Nounoua, Côte d'Ivoire (Afrique de l'Ouest). Int. J. Biol. Chem. Sci., 7(6): 2248-2263. DOI: http://dx.doi.org/10.4314/ijbcs.v7i6.6

Lavoué S. 2011. A new species of Petrocephalus Marcusen 1854 (Osteoglossomorpha: Mormyridae) from the Sanaga River basin, Cameroon. Zootaxa, 2934 : 20-28. DOI: http://dx.doi.org/10.11646/zootaxa.2934. 1.2

Lévêque C. 2006. Les introductions d'espèces dans les milieux naturels et leurs conséquences. In Les Poissons d'Eaux Continentales Africaines : Diversité, Ecologie, Utilisation par l'Homme, Lévêque C, Paugy D (eds). IRD : Paris ; 382 - 394.
Lévêque C, Paugy D. 2006. Impacts des activités humaines. In Les Poissons d'Eaux Continentales Africaines : Diversité, Ecologie, Utilisation par l'Homme, Lévêque C, Paugy D (eds). IRD : Paris ; 395 - 413.

Lienou G, Mahe G, Olivry JC, Naah E, Servat E, Sigha-Nkamdjou L, Sighomnou D, Ndam Ngoupayou J, Ekodeck GE, Paturel JE. 2005. Régimes des flux des matières solides en suspension au Cameroun : revue et synthèse à l'échelle des principaux écosystèmes ; diversité climatique et actions anthropiques. Hydrological Sciences Journal, 50(1): 110-123.

DOI: https://doi.org/10.1623/hysj.50.1.111.56 329

Magnet C, Pouomogne V. 2005. Etude environnementale $d u$ barrage de Lom Pangar. Thème 7 : étude de la pêche. MINEE : Yaoundé.

MINFOF. 2007. Convention de partenariat du 18 Janvier 2007 relative au développement d'une expérience pilote de gestion durable de la pêche le long du fleuve Djerem. Ministère des Forêts et de la Faune : Yaoundé.

Moelants T. 2010. The IUCN Red List of Threatened Species 2010: e.T182097A7797189. DOI: http://dx.doi.org/10.2305/IUCN.UK.201 0-3.RLTS.T182097A7797189.en.

Montchowui E, Chikou A, Kogbeto MJ, Lalèyè P. 2008. Biodiversité et structure des communautés de poissons du Lac Hlan au Benin. Int. J. Biol. Chem. Sci., 2 (2): 196206.

DOI: http://dx.doi.org/10.4314/ijbcs.v2i2.3973 3

Olivry JC. 1986. Fleuves et rivières du Cameroun. Monographies Hydrologiques. ORSTOM : Paris.

Onana JM. 2018. Cartographie des écosystèmes du Cameroun. Int. J. Biol. Chem. Sci., 12(2): 940-957. DOI: 
https://dx.doi.org/10.4314/ijbcs.v12i2.25

OREAD-BRECHE, 2011. Reformulation de l'évaluation environnementale et sociale du barrage de Lom Pangar. Etat des habitats aquatiques et projet de gestion des pêches dans la retenue. MINEE: Yaoundé.

Paugy D, Lévêque C, Teugels GG. 2003. Poissons d'Eaux Douces et Saumâtres de l'Afrique de l'Ouest. Tomes 1 et 2. IRD, MRAC : Paris, Tervuren.

Paugy D, Lévêque C. 2006. Régimes alimentaires et réseaux trophiques. In Les Poissons d'Eaux Continentales Africaines : Diversité, Ecologie, Utilisation par l'Homme, Lévêque C, Paugy D (eds). IRD : Paris ; 191 - 215.

Sonny D, Knudsen FR, Engers PS, Kvernstuen T, Sand O. 2006. Reactions of Cyprinids to infrasound in a lake and at the cooling water inlet of a nuclear power plant. Journal of Fish Biology, 69: 735-748. DOI: https://doi.org/10.1111/j.10958649.2006.01146.x

Stiassny MLJ, Teugels GG, Hopkins CD. 2007. Poissons d'Eaux Douces et Saumâtres de Basse Guinée, Ouest de l'Afrique Centrale. IRD, MRAC, AMNH: Paris, Tervuren, New York.
Tchawa P. 2012. Le Cameroun : une "Afrique en miniature »? Les Cahiers d'OutreMer, 259: 319-338. DOI: https://doi.org/10.4000/com.6640

Ter Braak CJF, Šmilauer P. 2012. Canoco reference manual and user's guide: software of ordination (version 5.0). Microcomputer Power: Ithaca, New York.

Vreven EJ, Musschoot MN, Snoeks J, Schliewen UK. 2016. The African hexaploidy Torini (Cypriniformes: Cyprinidae): review of a tumultuous history. Zoological Journal of the Linnean Society, 177 (2): 231-305. DOI: https://doi.org/10.1111/zoj.12366.

Vreven EJ, Musschoot T, Boden G, Stiassny MLJ. 2007. Espèces étrangères ou introduites en basse Guinée. In Poissons d'Eaux Douces et Saumâtres de Basse Guinée, Ouest de l'Afrique Centrale, Stiassny M, Teugels G, Hopkins C (eds). IRD, MRAC, AMNH: Paris, Tervuren, New York.

Zhang Z, Grabchak M. 2016. Entropic representation and estimation of diversity indices, Journal of Nonparametric Statistics, 28(3): 563-575. DOI: $10.1080 / 10485252.2016 .1190357$ 